\title{
Thromboembolies veineuses sous contraceptifs oraux combinés - etat des lieux
}

\author{
Depuis mai dernier, les médias ont régulièrement évoqué les thromboses veineuses \\ et les embolies pulmonaires survenues chez des femmes prenant des contraceptifs \\ oraux combinés $(\mathrm{COC})$. Or selon de récentes études, le risque de thromboembolies \\ veineuses (TEV) lié aux $\mathrm{COC}$ contenant de la drospirénone se situe, si l'on effectue \\ une comparaison directe, entre celui des $\operatorname{COC}$ de $2^{\text {ème }}$ et de $3^{\text {ème }}$ générations. Par \\ ailleurs, le risque de TEV est le plus élevé pendant la première année d'utilisation \\ d'un COC. Soulignons enfin que dans près de $40 \%$ des annonces spontanées de TEV \\ en provenance de Suisse, au moins un facteur de risque était présent. Aussi une \\ anamnèse (familiale) minutieuse et régulière est-elle indispensable.
}

\section{Christian De Geytera, \\ Christoph R. Meier ${ }^{b}$, \\ Danijela Pavelic Ferrettic, \\ Hiu Ying Kwanc, \\ Rudolf Stollerc} Endocrinologie gynécologique et médecine de la reproduction de l'Hôpital universitaire de Bâle, Clinique gynécologique

b Prof. Dr, responsable de la «Basel Pharmacoepidemiology Unit» et pharmacien chef de la pharmacie hospitalière de l'Hôpital universitaire de Bâle

c Swissmedic, Institut suisse des produits thérapeutiques, division Sécurité des médicaments, Berne

\footnotetext{
* Abréviations:

COC contraceptifs oraux combinés

TEV thromboembolie veineuse

TVP thrombose veineuse profonde

IC intervalle de confiance
}

Correspondance:

Dr Danijela Pavelic Ferretti PhD Medical Reviewer

Swissmedic

Hallerstr. 7

CH-3000 Berne

danijela.pavelic@swissmedic.ch
En mai 2009, les médias suisses se sont largement fait l'écho du cas d'une jeune fille sous COC* contenant de la drospirénone, qui a été victime d'une embolie pulmonaire centrale ayant entraîné des lésions cérébrales hypoxiques sévères. Malheureusement, le sujet a parfois été traité de manière incomplète et/ou déformée. Le 29 mai et le 11 juin 2009, Swissmedic a publié sur son site web des communiqués rappelant les faits principaux relatifs aux risque de TEV. Avec le concours de son «Human Medicines Expert Committee», Swissmedic a analysé les données et études les plus récentes sur le risque de TEV. Les conclusions, recommandations et mesures prévues sont détaillées ci-après.

Les risques cardiovasculaires de la pilule contraceptive ont été mis au jour peu après leur introduction sur le marché, au début des années 1960. Mais la réduction de la dose d'œstrogènes a par la suite permis de diminuer la fréquence des complications artérielles ischémiques ainsi que le risque de TEV. Les COC de première génération, avec $\geq 50 \mu \mathrm{g}$ d'œstrogènes, sont encore utilisés aujourd'hui, par exemple en association avec des inducteurs enzymatiques. Les COC récents contiennent moins de $50 \mu \mathrm{g}$ d'œstrogènes (micropilules) et sont classés en fonction de

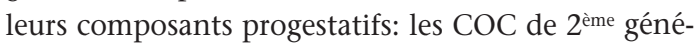
ration contiennent du lévonorgestrel, ceux de $3^{\text {ème }}$ génération du désogestrel ou du gestodène. Quant aux «COC antiandrogéniques», ils contiennent les nouveaux progestatifs que sont le chlormadinone et la drospirénone ou l'acétate de cyprotérone, utilisé depuis très longtemps. Enfin, les «minipilules» ont pour seul principe actif un composant progestatif.

En 1995/1996, trois nouvelles études épidémiologiques ([1, 2, 3]) ont montré que le risque de TEV dépend non seulement de la dose d'œstrogène, mais également du composant progestatif. Par ailleurs, les COC de $3^{\text {ème }}$ génération, qui étaient à l'époque nou- veaux, sont associés à un risque de TEV plus élevé que les pilules plus anciennes de $2^{\text {ème }}$ génération. Bien que rare, la survenue d'une TEV, effet indésirable potentiellement mortel, a alors fait l'objet de débats nourris dans les médias («pill scare») et les mises en garde énoncées dans l'information destinées aux patients et dans l'information professionnelle ont été élargies. Depuis 1998, des publications ont régulièrement rappelé le risque accru de TEV chez les patientes qui prennent des $\mathrm{COC}$ de 3 ème génération et chez les nouvelles utilisatrices de COC $[4,5,6]$. Enfin, plusieurs études d'observation ont confirmé que le taux de thromboembolies avec les COC de la $3^{\text {ème }}$ génération est augmenté d'un facteur 1,5 à 2 [7].

\section{Thromboembolies veineuses}

Les TEV se manifestent principalement sous deux formes: la thrombose veineuse profonde des membres inférieurs ou du bassin (TVP), associée ou non à une embolie pulmonaire. Le risque de TEV augmente avec l'âge et est plus élevé chez les femmes que chez les hommes. Par ailleurs, dans 1 à $2 \%$ des cas, les TEV ont une issue fatale. Les principaux facteurs de risque dans la population générale sont (a) une hospitalisation prolongée / opération, (b) l'existence d'une maladie maligne, (c) une infection et (d) la pose d'un cathéter veineux central [8]. Les études d'observation ont en outre montré que l'incidence annoncée des TEV dépend grandement de la précision du diagnostic et varie donc d'une étude à une autre [9]. En effet, à la phlébographie couramment pratiquée par le passé, on préfère aujourd'hui l'échographie pour diagnostiquer la TVP et la tomographie assistée par ordinateur pour l'embolie pulmonaire. Malgré des techniques diagnostiques plus fines, le recensement depuis plus de dix ans de l'incidence des TEV dans douze hôpitaux (Worcester, Etats-Unis) n'a pas mis en évidence 
d'augmentation significative du nombre de cas de cette affection $[8,10]$. L'incidence en fonction de l'âge des cas de TEV est de 11,4 cas pour 10000 personnes/an (intervalle de confiance [IC] 95\%: 10,8-12,0).

Le risque de TEV est accru chez les jeunes femmes prenant des COC. Les incidences de TEV varient selon des études, par exemple établies par une vaste méta-analyse [9] et une étude de cohorte récente [11] (voir tableau 1 ci-dessous). Dans les pays développés, les principales causes de mortalité maternelle liée à la grossesse et à l'accouchement sont, outre les complications liées aux mesures obstétriques, les TEV.

\begin{tabular}{|c|c|c|}
\hline \multicolumn{3}{|c|}{$\begin{array}{l}\text { Incidence des TEV chez les jeunes femmes selon les } \\
\text { études [9] et [11] }\end{array}$} \\
\hline & $\begin{array}{l}\text { Incidence de TEV pour } \\
10000 \text { années-femmes ( } 95 \% \text { IC) }\end{array}$ & $\begin{array}{l}\text { Réf. } \\
\text { biblio. }\end{array}$ \\
\hline $\begin{array}{l}\text { Femmes } \\
\text { sans COC }\end{array}$ & $1,9(1,2-2,5)$ & [11] \\
\hline $\begin{array}{l}\text { Femmes } \\
\text { avec COC }\end{array}$ & $\begin{array}{l}2,1(0,8-5,2) \\
7,4(5,2-9.6)\end{array}$ & $\begin{array}{l}{[9]} \\
{[11]}\end{array}$ \\
\hline $\begin{array}{l}\text { Femmes } \\
\text { enceintes }\end{array}$ & $10,3(5,5-17,7)$ & [11] \\
\hline
\end{tabular}

Thrombophilies: Chez les jeunes femmes, le risque de TEV sous COC est particulièrement élevé si elles présentent une prédisposition génétique, telle qu'une mutation hétérozygote du facteur $\mathrm{V}$ de Leiden (prévalence: $5 \%$ ), un déficit en antithrombine, un déficit en protéine $S(2 \%)$, une mutation G20210A du gène de la prothrombine (2\%) et un déficit en protéine $\mathrm{C}$ $(0,03 \%)$ [12]. L'évaluation du risque individuel d'une jeune femme est faite lors de la première prescription de COC ou en début de grossesse, non pas par des analyses de laboratoire, mais par une anamnèse familiale. Or, parce que celle-ci est souvent lacunaire, il serait souhaitable qu'elle soit refaite et approfondie. Par ailleurs, un dépistage systématique d'une anomalie génétique à l'origine d'une thrombophilie serait aujourd'hui techniquement possible, mais il est en général déconseillé pour des raisons de coûts pour le système de santé [12]. Autre motif invoqué: les conséquences psychologiques pour les personnes concernées en cas de découverte d'une mutation génétique [14]. Enfin, il paraît que même lorsque l'existence d'une thrombophilie est connue des médecins traitants, ils n'en tiennent pas suffisamment compte [15].

\section{Annonces spontanées en Suisse}

Les annonces spontanées permettent d'enregistrer précocement des signaux de sécurité, c'est-à-dire de possibles nouveaux risques. Comme elles ne permettent pas de déterminer la fréquence des effets indésirables et encore moins d'en tirer des conclusions comparatives, il est indispensable de se fonder sur des études épidémiologiques pour pouvoir faire des comparaisons fiables entre les groupes de préparations (cf. infra). Les effets indésirables des nouvelles préparations sont rapportés plus fréquemment que ceux des médicaments connus de longue date. Une plus grande prise de conscience de l'importance des annonces et l'augmentation massive de la fréquence des annonces des dernières années a conduit à la saisie d'annonces portant de préférence sur de nouvelles préparations.

Depuis la création du Centre de pharmacovigilance en 1990 et jusqu'au 2.6.2009, Swissmedic a reçu 1460 annonces spontanées relatives à des contraceptifs hormonaux (y c. COC, progestatifs seuls et implants à effet systémique). Pendant cette période, 120 cas de TEV ont été annoncés (cf. tableau 2).

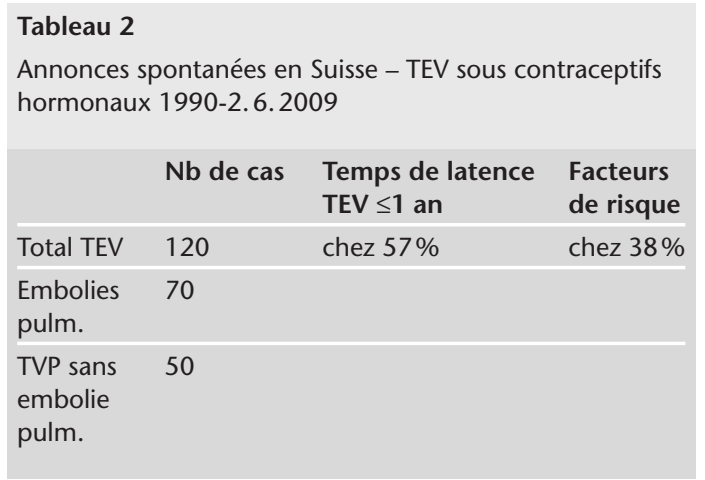

La grande majorité des cas de TEV (87\%) étaient associés à des COC antiandrogéniques et à des COC de $3^{\text {ème }}$ génération. Si l'on tient compte du «Reporting Ratio» (proportion d'annonces de TEV par rapport au nombre total d'annonces liées à des contraceptifs hormonaux), qui dépend moins de la fréquence d'annonce et de l'année de l'annonce, les COC avec drospirénone arrivent derrière ceux de $3^{\text {ème }}$ génération.

Dans près de $40 \%$ des cas de TEV, au moins un facteur de risque a pu être identifié. $57 \%$ des annonces de TEV ont été faites au cours de la première année d'utilisation, et la moitié d'entre elles au cours des trois premiers mois qui ont suivi le début de la contraception hormonale.

Jusqu'au 30.9.2009, Swissmedic a reçu au total dix annonces d'embolies pulmonaires ayant entraîné un décès mis en relation avec des contraceptifs hormonaux, c'est-à-dire avec des COC antiandrogéniques (acétate de cyprotérone [n=3], drospirénone [n=2], chlormadinone $[\mathrm{n}=1]$ ), des $\mathrm{COC}$ de $3^{\text {ème }}$ génération (désogestrel $[\mathrm{n}=3]$ ) et des progestatifs seuls (désogestrel $[\mathrm{n}=1])$, un groupe de préparations qui, au vu des connaissances actuelles, n'augmente pas le risque de TEV. La première a eu lieu en 1991 et la dernière en septembre 2009. Les femmes avaient entre 18 et 49 ans, et six d'entre elles avaient moins de 30 ans. Enfin, cinq femmes présentaient au moins un facteur de risque de TEV (surpoids, âge élevé, prédisposition familiale aux thromboses, vols de longue durée).

\section{Etudes épidémiologiques}

Quatre études épidémiologiques très récentes ont analysé le risque de TEV des COC contenant de la drospirénone (COC-D) et l'ont comparé à celui d'autres 
contraceptifs hormonaux $[16,17,18,19]$. Trois études sont des analyses rétrospectives de dossiers médicaux $[16,18,19]$, une est une étude prospective multicentrique [17] dans laquelle les patientes donnaient initialment elles-mêmes des informations sur les diagnostics et sur les expositions en répondant à un questionnaire.

Les auteurs de l'étude européenne EURAS [17] et ceux de l'étude américaine INGENIX [16] sont tous parvenus à la conclusion que les COC-D n'entraînent pas de risque supérieur aux autres COC. Ces deux études ont inclus, outre des cas idiopathiques, également des cas présentant des facteurs de risque directs, tels qu'un trauma récent ou une opération chirurgicale. Ceci réduit la possibilité de mesurer l'effet des COC sur le risque de TEV et tend à minimiser une éventuelle augmentation du risque induit par les COC. L'étude INGENIX a par ailleurs comparé le risque de TEV chez des femmes prenant des COC-D avec celui d'un groupe témoin composé de femmes qui prenaient «d'autres COC», sans que ces derniers ne soient plus précisément définis. Malgré ce point faible qui s'ajoute à d'autres insuffisances de ces études, il est possible d'en conclure avec prudence que le risque de TEV sous COC-D est du même ordre que celui d'autres COC. En revanche, on ignore toujours si ce risque doit être considéré comme relativement faible et comparable à celui

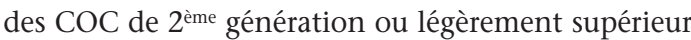
à l'instar de celui associé aux COC de 3 ème génération.

Deux nouvelles études épidémiologiques réalisées aux Pays-Bas [18] et au Danemark [19] et publiées en août 2009 faisaient en revanche état d'un risque de TEV accru avec les COC-D, le risque relatif de TEV se situant entre les COC de $2^{\text {ème }}$ et de $3^{\text {ème }}$ générations. Les auteurs de l'étude cas-contrôles [18] et de l'étude de cohorte [19] ont également inclus, comme dans l'étude EURAS des femmes qui ne prenaient pas de COC et ont comparé le risque de TEV sous différents COC à celui des femmes qui ne prenaient pas de COC. Les résultats étaient globalement cohérents et prouvaient d'une part que le risque de TEV augmente avec l'âge de la femme et avec la teneur en œstrogènes de la pilule, et d'autre part que le risque de TEV est le plus élevé au début du traitement et qu'il diminue par la suite. La comparaison directe des différents COC a une nouvelle fois montré que les COC de 3 ème génération présentent une risque de TEV presque deux fois

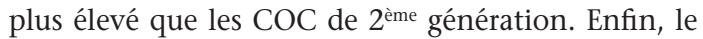
risque de TEV sous COC-D était inférieur à celui lié aux $\mathrm{COC}$ de $3^{\text {ème }}$ génération, mais supérieur à celui as-

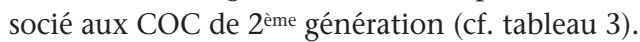

Ces études sont limitées par le fait que certains facteurs de risque, tels que le surpoids, ou les antécédents familiaux n'ont pas été considérés, et par la largeur des intervalles de confiance. Par ailleurs dans l'étude danoise la question d'une sous-estimation du risque de TEV des COC de $2^{\text {ème }}$ génération reste ouverte.

En résumé, ces quatre études épidémiologiques démontrent une nouvelle fois que les femmes qui prennent des COC ont globalement un risque de TEV plus élevé que celles qui n'en prennent pas, ce constat étant également vrai pour les COC-D. En ce qui concerne le risque de TEV lié aux COC-D, il apparaît, si l'on fait une comparaison directe, qu'il se situe

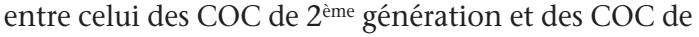
$3^{\text {ème }}$ génération.

\section{Recommandations et mesures}

Anamnèse familiale / tests sanguins: Chez les jeunes utilisatrices de COC, des TEV se produisent le plus fréquemment chez celles qui présentent une prédisposition génétique (thrombophilie) ou chez les femmes qui sont atteintes du syndrome des antiphospholipides. Ainsi le risque de TEV est 20 fois supérieur chez les femmes présentant une mutation du facteur $\mathrm{V}$ de Leiden. Le syndrome des antiphospholipides est une maladie auto-immune caractérisée par une tendance accrue aux thromboses et des fausses-couches répétées. En général, le syndrome des antiphospholipides apparaît souvent dans un tableau clinique de lupus érythémateux. Tant le syndrome des antiphospholipides que la thrombophilie héréditaire peuvent être dépistés par une anamnèse et représentent des contreindications à la prescription d'un COC. En cas de suspicion de ces anomalies génétiques au vu de l'anamnèse, le diagnostic peut être confirmé par des analyses de laboratoire.

Prescription de COC: Les COC ne doivent être prescrits qu'après détermination minutieuse de l'indica-

Tableau 3

Risque de TEV lié aux COC contenant de la drospirénone comparé à ce même risque associé aux autres COC

\begin{tabular}{|c|c|c|c|c|c|}
\hline & $\begin{array}{l}\text { Groupe de } \\
\text { préparations }\end{array}$ & $\begin{array}{l}\text { Etude } \\
\text { EURAS* [17] }\end{array}$ & $\begin{array}{l}\text { Etude } \\
\text { INGENIX** [16] }\end{array}$ & $\begin{array}{l}\text { Etude } \\
\text { hollandaise }^{* * *}[18]\end{array}$ & $\begin{array}{l}\text { Etude } \\
\text { danoise }^{* *}[19]\end{array}$ \\
\hline $\mathrm{COC}$ antiandrogéniques & DRS & 1,0 (référence) & $1,0(0.5-1.9)$ & $6,3(2,9-13,7)$ & $1,64(1,27-2,10)$ \\
\hline COC $2^{\text {ème }}$ génération & LEV & $1,0(0.6-1.8)$ & -- & $3,6(2,9-4,6)$ & 1,0 (référence) \\
\hline \multirow[t]{2}{*}{$\mathrm{COC} 3^{\text {ème }}$ génération } & GES & -- & -- & $5,6(3,7-8,4)$ & $1,86(1,59-2,18)$ \\
\hline & DES & -- & -- & $7,3(5,3-10,0)$ & $1,82(1,49-2,22)$ \\
\hline «Autres CO» & & -- & 1,0 (référence) & -- & -- \\
\hline Sans CO & & -- & -- & 1,0 (référence) & -- \\
\hline
\end{tabular}

Toutes les valeurs sont ajustées; $(\ldots)=95 \%$ intervalle de confiance; * Hazard ratio; ** Rate ratio; *** Odds ratio; DRS = drospirénone; $\mathrm{LEV}$ = lévonorgestrel; GES = gestodène; DES = désogestrel. 
tion. Lors de la prescription de COC-D et de préparations de $3^{\text {ème }}$ génération, il convient de prendre particulièrement en compte le risque de TEV. Si la patiente présente un facteur de risque, il convient d'envisager une méthode contraceptive non hormonale. Les COCD doivent être utilisés essentiellement lorsque l'effet partiel antiandrogénique du progestatif qu'est la drospirénone est souhaité pour des raisons thérapeutiques, c.-à-d. à des fins contraceptives chez des femmes souffrant d'hirsutisme ou d'acné. Ajoutons qu'il est fortement déconseillé de fumer, afin de limiter les risques de complications artérielles.

Information professionnelle et information destinée aux patients: L'information sur le médicament énonce en détail les mesures de précaution à observer. Quant à l'actuelle médiatisation de ce dossier, elle montre qu'il est souhaitable que les utilisatrices lisent elles aussi attentivement l'information sur le médicament et qu'elles posent leurs éventuelles questions à leur médecin.

Embolies pulmonaires: Du fait de sa symptomatologie non spécifique, il est difficile de poser précocement un diagnostic d'embolie pulmonaire. Les signes les plus fréquents sont une performance physique, une dyspnée et des syncopes. En particulier, si des jeunes femmes se présent avec des signes pleuraux, il faudrait penser à leur demander si elles prennent des COC et considérer l'embolie pulmonaire comme diagnostic différentiel.

Mesures introduites par Swissmedic: Dans le cadre de la réévaluation en cours de tous les COC, Swissmedic a ouvert une «procédure de révision» qui compare toutes les préparations contenant de la drospirénone aux autres COC. Les principaux résultats des nouvelles études seront en outre intégrés dans leurs informations respectives.

Annonce d'effets indésirables suspectés: Pour que Swissmedic puisse poursuivre la surveillance du risque de TEV associé à la prise quotidienne de médicaments, vos annonces lui sont indispensables. C'est pourquoi un formulaire jaune prévu à cet effet a été inséré dans le présent cahier. Mais vous pouvez aussi le télécharger sur le site de Swissmedic www.swissmedic.ch $(\rightarrow$ «Accès direct à» $\rightarrow$ «Annonces d'effets indésirables») ou le trouver en dernière page du Compendium suisse des médicaments.

\section{Références}

1 Jick H, Jick SS, Gurewich V, Myers MW, Vasilakis C. Risk of idiopathic cardiovascular death and non-fatal venous thromboembolism in women using oral contraceptives with differing progestagen components. Lancet. 1995;346:1589-93.

2 Effect of different progestagens in low oestrogen oral contraceptives on venous thromboembolic disease. World Health Organization Collaborative Study of Cardiovascular Disease and Steroid Hormone Contraception. Lancet. 1995;346:1582-8.

3 Spitzer WO, Lewis MA, Heinemann LAJ, Thorogood M, MacRae KD. Third generation oral contraceptives and risk of venous thromboembolic disorders: an international casecontrol study. BMJ. 1996;312:83-8.
4 De Bruijn SF, Stam J, Vandenbroucke JP. Increased risk of cerebral venous sinus thrombosis with third-generation oral contraceptives. Cerebral Venous Sinus Thrombosis Study Group. Lancet. 1998;351(9113):1404.

5 Jick SS, Vasilakis C, Jick H. Pregnancies and terminations after 1995 warning about third-generation oral contraceptives. Lancet. 1998;351:1404-5.

6 Herings RMC, Urquhart J, Leufkens HGM. Venous thromboembolism among new users of different oral contraceptives. Lancet. 1999;354:127-8.

7 Maitra NN, Kulier R, Bloemenkamp K, Helmerhorst FM, Gülmezoglu AM. Progestogens in combined oral contraceptives for contraception. 2004; Issue 3, CD004861.

8 Spencer FA, Emery C, Joffe SW, Pacifico L, Lessard D, Reed G, et al. Incidence rates, clinical profile, and outcome of patients with venous thromboembolism. The Worcester VTE study. J Thromb Thrombolysis. 2009; Jul 24. [Epub ahead of print].

9 Gomes MPV, Deitcher SR. Risk of venous thromboembolic disease associated with hormonal contraceptives and hormone replacement therapy. Arch Intern Med. 2004;164:1965-76.

10 Spencer FA, Emery C, Lessard D, Anderson F, Emani S, Aragam J, et al. The Worcester Venous Thromboembolism study. A population-based study of the clinical epidemiology of venous thromboembolism. J Gen Intern Med. 2006;21:722-7.

11 Samuelsson E, Hägg S. Incidence of venous thromboembolism in young Swedish women and possibly preventable cases among combined oral contraceptive users. Acta Obstet Gynecol Scand. 2004;83:674-81.

12 Andreassi MG, Botto N, Maffei S. Factor V Leiden, prothrombin G20210A substitution and hormone therapy: indications for molecular screening. Clin Chem Lab Med. 2006;44:514-21.

13 Wu O, Robertson L, Twaddle S, Lowe GD, Clark P, Greaves M, et al. Screening for thrombophilia in high-risk situations: systematic review and costeffectiveness analysis. The Thrombosis: Risk and Economic Assessment of Thrombophilia Screening (TREATS) study. Health Technol Assess. 2006;10:1-110.

14 Gartner V, Weber M, Eichinger S. The emotional impact of genetic testing and aspects of counseling prior to prescription of oral contraceptives. Contraception. 2008;78:392-398.

15 Dunn N, White I, Freemantle S, Mann R. The role of prescribing and referral bias in studies of the association between third generation oral contraceptives and increased risk of thromboembolism. Pharmacoepidemiol Drug Saf. 1998;7(1):3-14.

16 Seeger JD, Loughlin J, Eng M, Clifford R, Cutone J, Walker AM. Risk of thromboembolism in women taking ethinylestradiol/drospirenone and other oral contraceptives. Obstet Gynecol. 2007;110:587-93.

17 Dinger JC, Heinemann LA, Kühl-Habich D. The safety of drospirenone-containing oral contraceptive: final results from the European Active Surveillance study on Oral Contraceptives based on 142,475 womenyears of observation. Contraception. 2007;75:344-54

18 van Hylckama Vlieg A, Helmerhorst FM, Vandenbroucke JP, Doggen CJ, Rosendaal FR. The venous thrombotic risk of oral contraceptives, effects of oestrogen dose and progestogen type: results of the MEGA case-control study. BMJ. 2009;339:b2921.

19 Lidegaard Ø, Løkkegaard E, Svendsen AL, Agger C. Hormonal contraception and risk of venous thromboembolism: national follow-up study. BMJ. 2009;339:b2890. 